\title{
Performance Evaluation of Efficient Routing Protocols in Delay Tolerant Network under Different Human Mobility Models
}

\author{
Namita Mehta ${ }^{1}$ and Mehul Shah ${ }^{2}$ \\ ${ }^{1}$ Faculty of Electronics \& Communication Engg. \\ $B \& B$ Institute of Technology,V.V.Nagar \\ ${ }^{2}$ Assistant Professor, Department of Communication Engg. \\ G.H.Patel College of Engineering \& Technology, V.V.Nagar \\ Gujrat, India. \\ uvmehta2000@yahoo.com,mehul_gcet@yahoo.com
}

\begin{abstract}
Delay and/or Disruption-Tolerant Networking (DTN) is a novel communication prototype that can span across multiple networks and deal with unpredicted conditions in the Internet model.Delay-tolerant networks (DTNs) are partitioned wireless ad hoc networks with intermittent connectivity. Additional terminology in this family of dynamic networks includes disruption-tolerant networks, intermittently connected networks, and opportunistic networks. Routing of the packets in DTN is based on store-carry-and forward paradigm. In this paper, we study and analyze performance of well known PROPHET and Spray and Wait routing protocol, under different human mobility models such as Truncated Levy Walk mobility model (TLW),Self-similar Least Action Walk (SLAW) and Random way point (RWP) model. The MATLAB simulator is used in order to analyze the performance of these routing protocols. Simulation results illustrate that Spray and Wait significantly outperforms the PROPHET on aspects of delivery ratio, average delay and communication overhead.
\end{abstract}

Keywords: Delay Tolerant Network, Routing Protocols,Human Mobility, Truncated Levy Walk model, SLAW model

\section{Introduction}

Delay Tolerant networks[1,2] , also referred to as Intermittently Connected Mobile Networks, in which at any given time instance, the probability of continuous end to end connectivity from source to destination is very low.In DTN the connectivity of the network is maintained by nodes only when they encounter with each other. A node stores the message inside the buffer until an appropriate communication opportunity arises. Nodes in DTN make use of a "store-carry-forward" paradigm [3],to communicate among themselves. In this Challenged Network every node acts as a relay node stores the arriving message inside the buffer, carries the message while moving and forward it when they encounter with other nodes.

The rest of paper is organized as follows. In Section 2 we mention PROPHET and Spray and Wait popular routing protocols in DTN considered for comparison. Section 3 describes mobility models. In Section 4 we discuss about the simulation setup and performance metrics. Section 5 displays simulation results and comparison based on different mobility models namely Turncated Levy Walk,Self-similar Least Action Walkand Random way point mobility models.Section 6concludes this paper. 


\section{Routing Protocol in DTN}

In DTN, there is no exists continual route between two end points as a results of high delay and sporadic disconnection. To handle the routing in this Opportunistic networkseveralsolutions have been proposed. Routing protocols plays a crucial role to distribute messages between two communicating nodes.

\subsection{PROPHET}

PROPHET, Probabilistic Routing Protocol using History of Encounters and Transitivity, is an evolution of Epidemic routing that commencewith concept of delivery predictability [4]. The basic assumption in the PROPHET is that node's mobility is not a random, but it is a repeating behavior. It means that in PROPHET it might be possible that node has visited a location several time. Due to this possibility, this pattern will be repeated in the future. In PROPHET every node uses probabilistic metric called delivery predictability to transfer messages to a reliable node. The higher delivery predictability for a node indicates that it is more reliable than other nodes to forward message to destination. The forwarding strategy of PROPHET is differing than Epidemic Routing. Routing protocol PROPHET proposed for improve the delivery probability and reduce the wastage of network resources in Epidemic routing.The operation of the PROPHET protocol could be calculated by delivery predictabilities and then forwarding strategies.

The calculations of delivery predictability of nodes are divided in to three parts. Nodes update their delivery predictability metrics when they encounter with each other. The calculation is shown below, where $P_{\text {init }} \in[0,1]$ is an initialization constant.

$$
\mathbf{P}_{(\mathrm{a}, \mathrm{b})}=\mathbf{P}_{(\mathrm{a}, \mathrm{b}) \text { old }}+\left(1-\mathbf{P}_{(\mathrm{a}, \mathrm{b}) \text { old }}\right) \times \mathbf{P}_{\text {init }}
$$

On the other hand, if nodes encounter with lower probabilities for a longer period of time, then the delivery predictability metric is updated by the nodes as shown below: where $\gamma \in[0,1]$ is an aging constant and $\mathrm{k}$ is the number of the time units that have elapsed since last time the metric was aged.

$$
\mathbf{P}_{(\mathbf{a}, \mathrm{b})}=\mathbf{P}_{(\mathbf{a}, \mathrm{b}) \mathbf{o l d}} \times \gamma^{\wedge} \mathbf{k}
$$

The delivery predictability has transitive property meaning that if node A frequently encounters node $\mathrm{B}$ and node $\mathrm{B}$ frequently encounters node $\mathrm{C}$, then node $\mathrm{C}$ probably is a good node to forward messages destined for node A. This equation shows how this transitivity affects the delivery predictability, where $\beta \in[0,1]$ is a scaling constant that decides how large impact the transitivity should have on the delivery predictability.

$$
\mathbf{P}_{(\mathrm{a}, \mathrm{c})}=\mathbf{P}_{(\mathrm{a}, \mathrm{c}) \text { old }}+\left(1-\mathbf{P}_{(\mathrm{a}, \mathrm{c}) \text { old }}\right) \times \mathbf{P}_{(\mathrm{a}, \mathrm{b})} \times \mathbf{P}_{(\mathrm{b}, \mathrm{c})} \times \boldsymbol{\beta}
$$

\subsection{Spray and Wait}

To control the level of spreading of messages throughout the network similar to epidemic routing[5], spray and wait [6,7] routing protocol is used. Flooding techniques are used to forward multiple copies of received messages. This algorithm works in two phases, namely the spray phase and other, the wait phase. In the spray phase, a node generates limited number of copies (L) of the packets which are spread over the networks by to relay nodes. At any instant, the node checks Lcopies of the message when it encounter the other nodes within transmission range. If Lhas the value higher than 1, then half (i.e. L / 2) copies of the messages are spray to its neighbor and update L. When only single copy left, a wait phase starts and direct transmission routing is used to forward single copy to the destination directly. Each $\mathrm{N}$ nodes carries a message copy to perform 
direct transmission of message until it successfully delivered the message to the destination.

\section{Mobility Model}

Human mobility has a significant influence on the performance of network systems that involve daily human activities. Therefore, studying and finding fundamental characteristics of human mobility and developing realistic human mobility models are essential for optimum construction of these systems.Mobility models follow the performance of real life mobile user as closely as possible.

\subsection{Truncated Levy Walk Mobility Model}

Work based on various real world traces and accomplished that the movement of real people from various outdoor settings follows a Levy Walk [8]. People move within a predefined (also confined) area for routine activities. These tendencies are not confined in common mobility models such as random way point (RWP). For a reliable assessment of routing protocols human mobility based TLW mobility model brings out in this article. As the mobile devices are often attached or controlled by human so, it is important to study human mobility model such as TLW model for accurately understanding nodes mobility. The mobile users having distinctiveness human walk in this model. Human walk characteristics followed by flight length, pause time and inter-contact time.

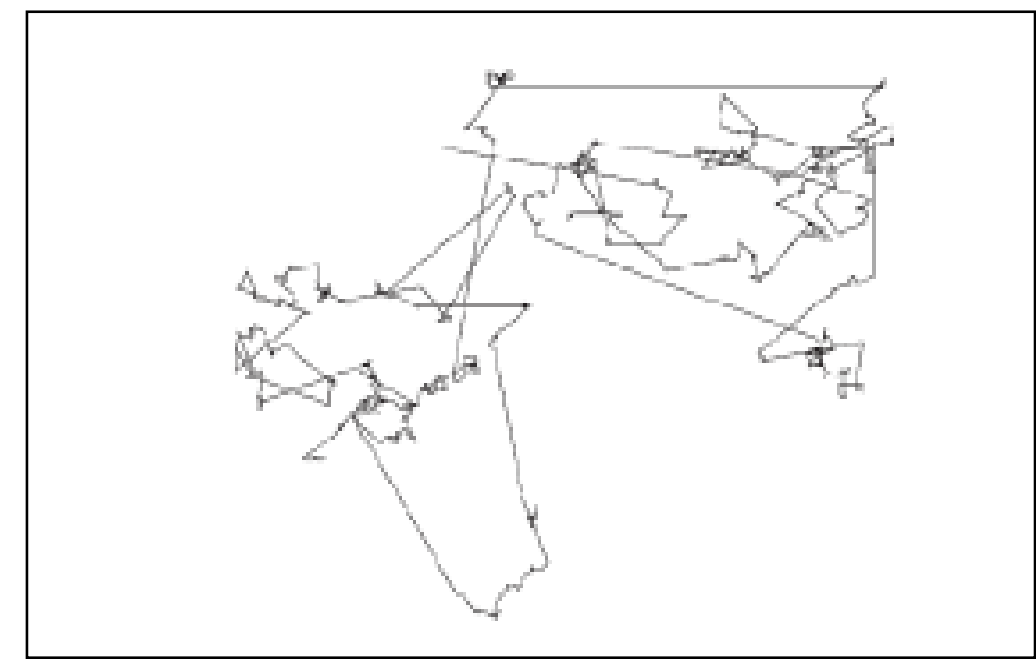

Figure 1. Levy Walk Mobility Model's Waypoints

Human walk characteristics followed by flight length, pause times, and intercontact time. These characteristics illustrate in Figure 1.

i) Probability Distribution Function of Flight lengths for Levy-Walk model is

Where $l_{\max }$ is the maximum flight length and

$$
\left.\begin{array}{c}
\rho(l) \alpha|l|^{-(1+\alpha)} ; 0<l<l_{\max } \\
; \quad 0<\alpha<2
\end{array}\right\}
$$

$\alpha$ is Levy exponent for flight length distribution

ii) Probability Distribution Function of Pause time for Levy-Walk model is 


$$
\left.\begin{array}{c}
\phi(t) \alpha t^{-(1+\beta)} ; 0<\mathrm{t}<\mathrm{tmax}(2) \\
; 0<\beta<2
\end{array}\right\}
$$

Where $t_{\max }$ is the maximum pause time and

$\beta$ is Levy exponent for pause time distribution.

iii) A uniform distribution of Turning angles within [0, 360].

iv) Velocity increases as flight lengths increase.

\subsection{Random Way Point}

Generally, in traditional networks frequently used mobility model is Random Waypoint mobility model. In Random waypoint movement model (RWPM), a MN (mobile node)choose a random location on a simulation area and a speed that is uniformly distributed with a minimum and maximum value from $[0, \mathrm{Vmax}]$. Where $\mathrm{V}_{\max }$ is the maximum velocity allowed for each node. When the node has arrived, it will wait for random amount of time, and then continue this process again [9].

\subsection{Self-similar Least Action Walk (SLAW)}

In [10], Lee et al. introduce a novel mobility model called SLAW (Self-similar Least Action Walk) based on different extent studies of human walk traces. SLAW stimulates more frequent and regular contacts among nodes that result in more predictable and shorter routing delays for those protocols. SLAW can be an important tool for emulating human walk behaviors in various application scenarios. These comprise inter-contact times, truncated power-law distributions of flights, pause time, diversely defined areas of individual mobility and fractal way-points. None of presented mobility models successfully captures every one of these characteristics. The statistical characteristics are summarized for Human mobility as given below.

- Truncated Power-Law Flights and Pause Time:

It is defined as the lengths of human flights which are characterize to be straight line trips without directional change or pause (or lines between two consecutive waypoints) has a truncated power-law distribution.

- Truncated Power-Law Inter-contact Times (ICTS):

The distribution of inter-contact times, means the times elapsed between two consecutive contacts of the same persons can also be represented by a truncated power law distribution which consists of a power-law head followed by an exponentially decomposing tail after a certain characteristic time.

- Heterogeneously Bounded Mobility Areas:

People frequently move within their own confined areas of mobility and different people may have usually different mobility areas.

- Fractal Waypoints:

While from the scrutiny of the GPS traces of human walks, we can say that the waypoints of humans can be represented by fractal points. This means that people are always more attracted to more popular places.

\section{Performance Metrics and Simulation Setup}

\subsection{Performance Metrics}

There are three metrics are used for the performance of the different routing protocols: 


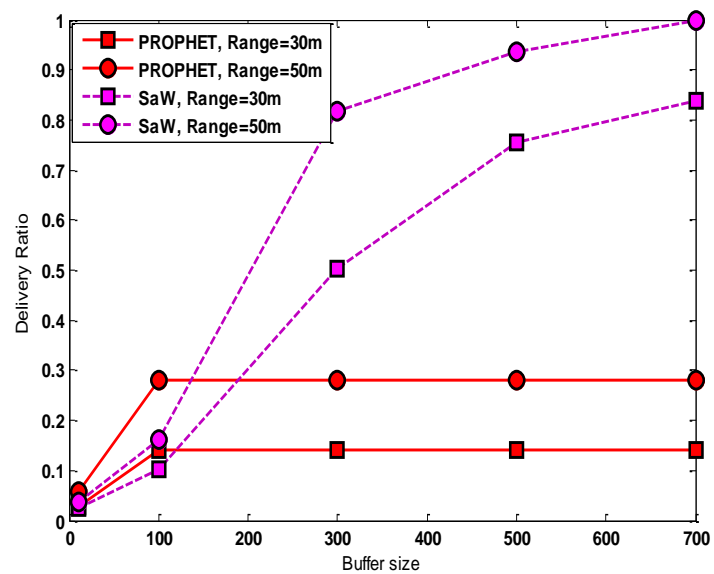

Figure 2.1 Impact of Varying Buffer Size on Delivery Ratio

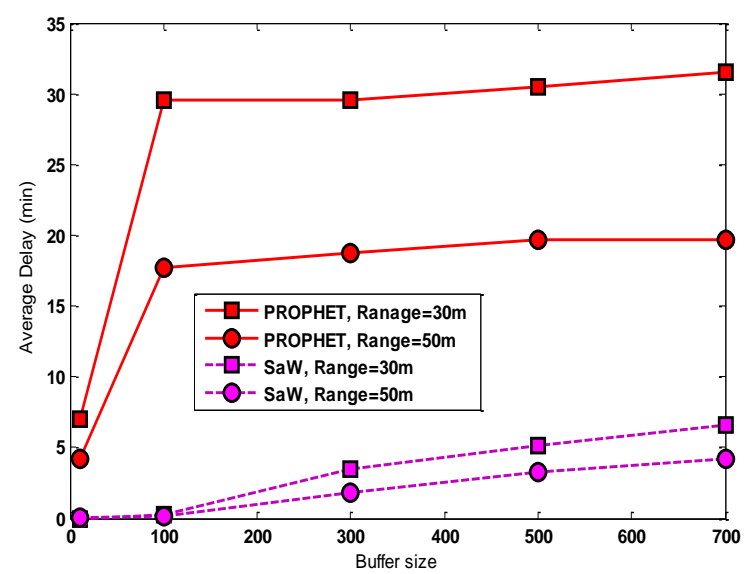

Figure 2.2 Impact of Varying Buffer Size on Average Delay

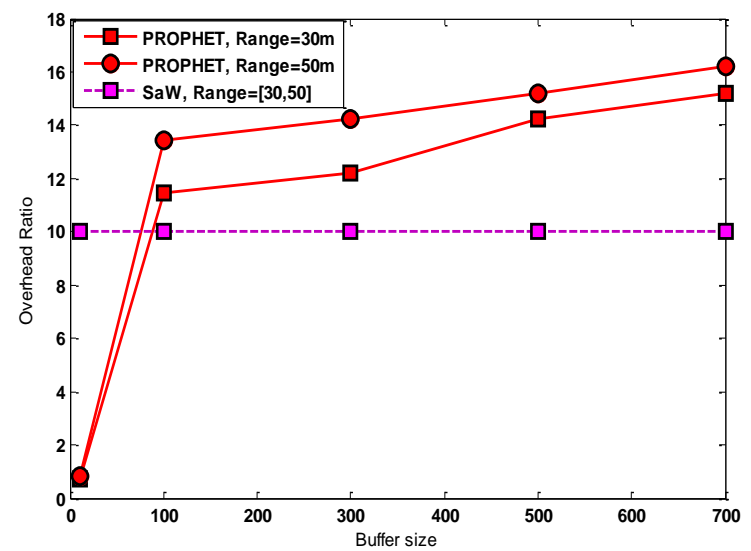

Figure 2.3 Impact of Varying Buffer Size on Overhead Ratio

Figure 2. Simulation Results from Impact of Varying Transmission Range on Random Way Point Mobility Model

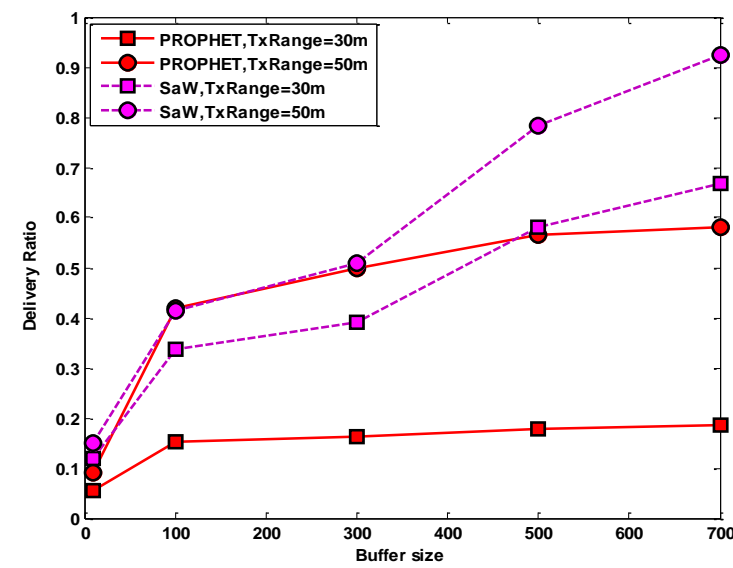

Figure 3.1 Impact of Varying Buffer Size on Delivery Ratio

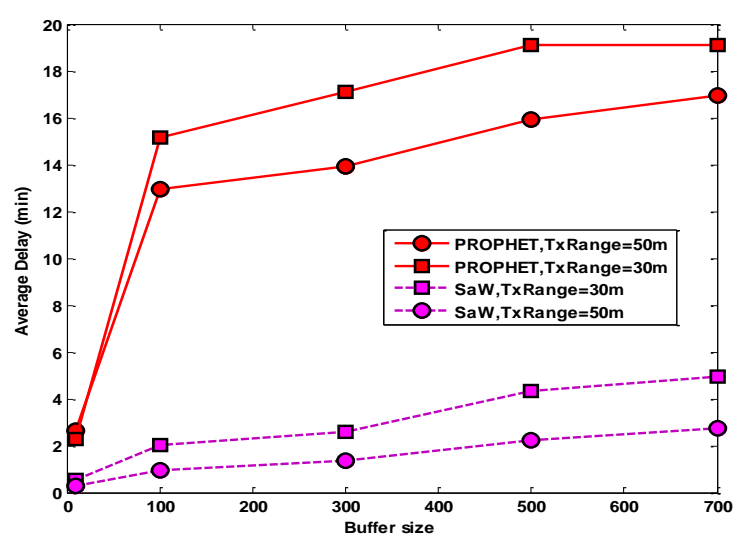

Figure 3.2 Impact of Varying Buffer Size on Average Delay

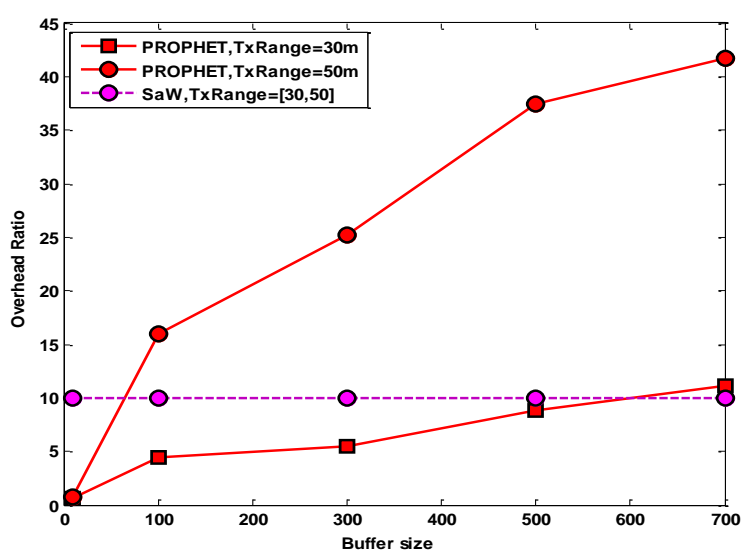

Figure 3.3 Impact of Varying Buffer Size on Overhead Ratio

Figure 3. Simulation Results from Impact of Varying Transmission Range on Truncated Levy Walk Mobility Model 


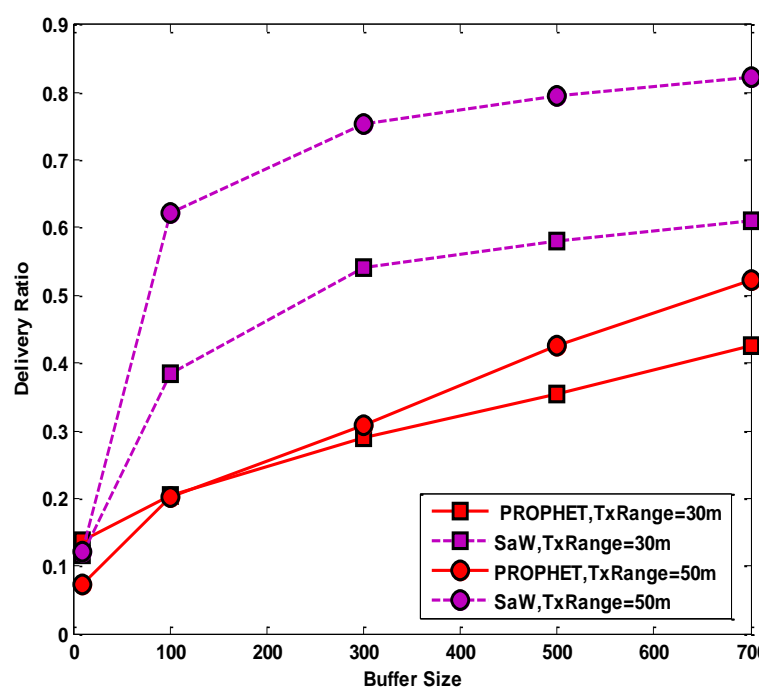

Figure 4.1 Impact of Varying Buffer Size on Delivery Ratio

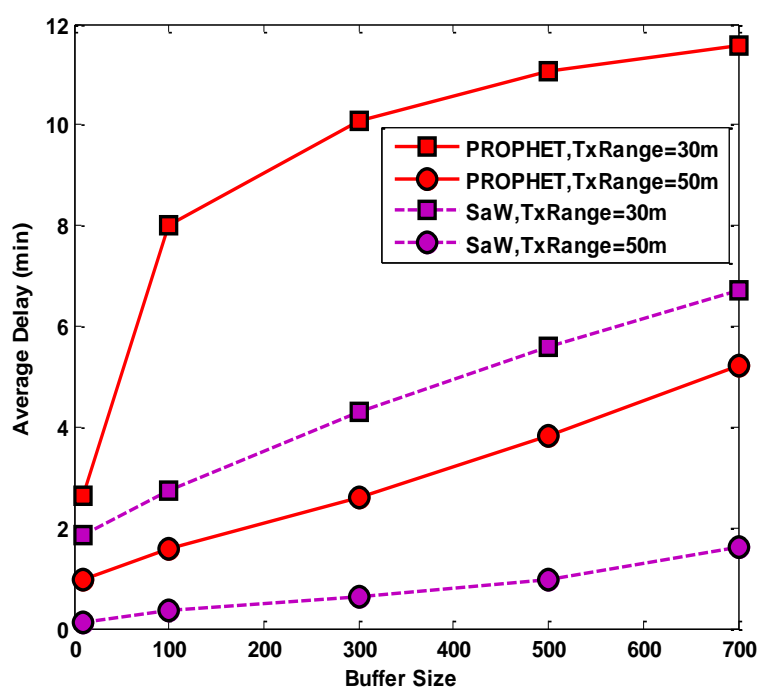

Figure 4.2 Impact of Varying Buffer Size on Average Delay

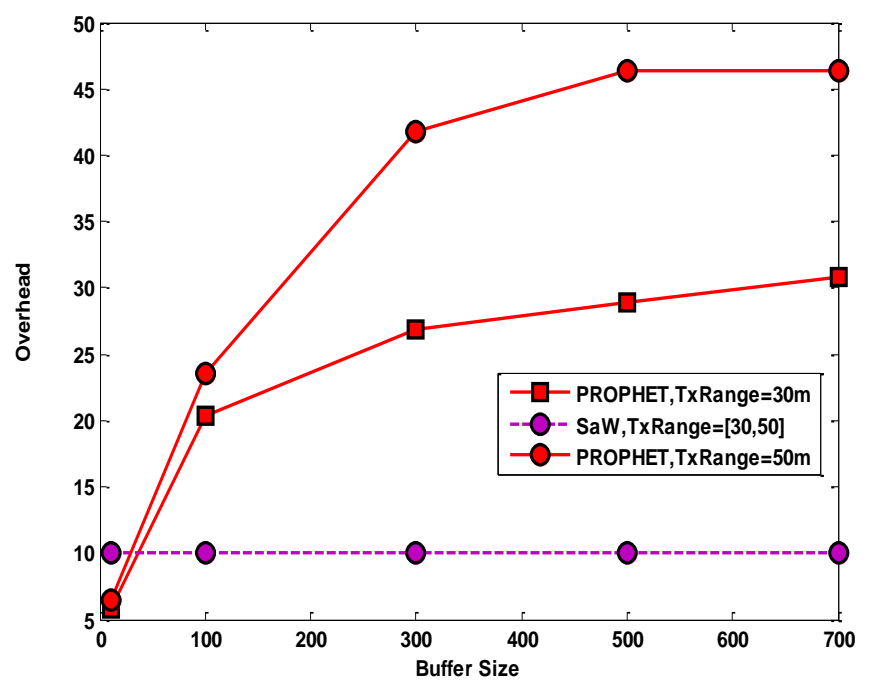

Figure 4.3 Impact of Varying Buffer Size on Overhead Ratio

Figure 4. Simulation Results from Impact of Varying Transmission Range on Self-similar Least Action Walk (SLAW) Model

copy. The overhead for Epidemic and PROPHET increases with increase in buffer size because more packets can be stored in the buffer and dropping of packets is reduced. Therefore, more packets are relayed through the network. PROPHET has lower communication overhead than Epidemic because PROPHET sends packets only to reliable nodes, while Epidemic forward packets to all possible nodes.

An additional observation from the graphs that increasing the transmission range usually increases the performance in terms of delivery ratio and average delay, but also increases the network overhead in PROPHET. It can be clearly seen that, the probability of meeting two nodes with each other increases with a larger communication range. Because it allows more nodes to directly communicate with larger number of other nodes. 


\section{Conclusion}

In this paper, we have studied and analyzed the performance of the routing protocols underthree human mobility models. The main goal is to determine the best routing protocol with different mobility models based on performance metrics such as delivery probability, average delay and communication overhead. Inter-contact times, truncated power-law distributions of flights, pause time, diversely defined areas of individual mobility and fractal way-points these features are captured by SLAW mobility model. None of presented mobility models successfully captures every one of these characteristics. In TLWmobility model, the routing protocol makes use of truncated power law distributions of pause time and flight length for human mobility. These parameters are not captured in common mobility model such as RWP. To reflect the reality SLAW model is more realistic than TLW and RWP models. Simulations performed have shown that in a human mobility based TLW model, SLAW model and RWP based scenario, Spray and Wait clearly gives better performance than PROPHET Routing protocol. Andcommunication overhead is much less for Spray and Wait as compared to PROPHET.

\section{References}

[1] K. Fall, "A delay-tolerant network architecture for challenged internet", Proc.ACM SIGCOMM, (2003).

[2] S. Jain, K. Fall and R. Patra, "Routing in a delay tolerant network", Proc. of ACM SIGCOMM, (2004).

[3] V. Cerf, S. Burleigh, A. Hooke, L. Torgerson, R. Durst, K. Scott, K. Fall and H. Weiss, "RFC 4838, Delay Tolerant Networking Architecture", IRTF DTN Research Group, (2007).

[4] A. Lindgren, A.Doria and O. Schelen, "Probabilistic routing in intermittently connected networks", ACM SIGMOBILE Mobile Computing and Communications Review, vol. 7, no. 3, (2003), pp. 19-20.

[5] A. Vahdat and D. Becker, "Epidemic routing for partially connected ad hoc networks", Duke University Durham, USA, Technical Report CS-2000-06, (2000).

[6] T. Spyropoulos, K. Psounis and C. S. Raghavendra, "Single copy routing in intermittently connected mobile networks", Proc. of IEEE Secon, (2004).

[7] T. Spyropoulos, K. Psounis and C. S. Raghavendra, "Spray and wait: Efficient routing in intermittently connected mobile networks", Proceedings of ACMSIGCOMM workshop on Delay Tolerant Networking (WDTN), (2005).

[8] I. Rhee, M. Shin, S. Hong, K. Lee, S. J. Kim and S. Chong, "On the levy-walk nature of human mobility”, IEEE/ACM Transactions on Networking, vol. 19, no. 3, (2011), pp. 630-643.

[9] T. Camp, J. Boleng and V. Davies, "A Survey of Mobility Models for Ad Hoc Network Research", Wireless Communication \& Mobile Computing (WCMC): Special issue on Mobile Ad Hoc Networking: Research, Trends and Applications,(2002), pp. 483-502.

[10] K. Lee, S. Hong, S. J. Kim, I. Rhee and S. Chong, "SLAW: A New Mobility Model for Human Walks", INFOCOM, IEEE, vol. 19-25, (2009), pp. 855 - 863.

\section{Authors}

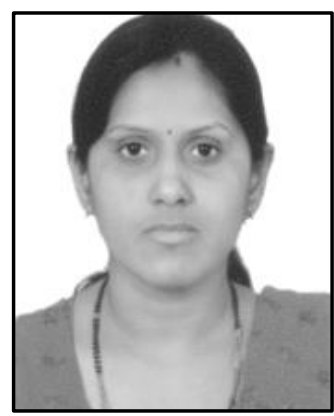

Namita Mehtais working as a Lecturer at the Department of Electronics \& Communication Engg, B \& B Institute of Technology, V.V.Nagar, Gujrat, India. I completed my B.E. in year 2005.My Master in Communication Engg. iscompleted from G.H.Patel College of Engg. \& Technology. 


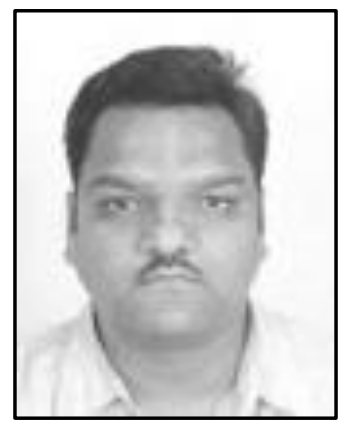

Dr. Mehul Shah Presently working as Assistant Professor in Electronics \& Communication Department, G.H.Patel College of Engg.\& Technology.

$\mathrm{He}$ is currently completing a Ph.D. from IIT Bombay, Electrical Engineering Department, India. He worked on Cell phone based Wireless Sensor Networks and actively involved in Indo-UK Next generation network project on Pervasive sensor Environment. His research interests are in Wireless Communication and Mobile Adhoc\& Sensor Networks. 
International Journal of Grid Distribution Computing Vol. 8, No. 1 (2015) 